\title{
Collaborative Translation in the Digital AGE*
}

\author{
YVONNE TSAI \\ National Taiwan University, Taiwan \\ yvtsai@ntu.edu.tw
}

\begin{abstract}
This study investigates the learning experiences of student translators participating in collaborative translation with and without the use of translation technology through collaborative translation teaching and practical exercises. The effectiveness and efficiency of two types of collaboration (face-to-face and virtual) are surveyed in terms of students' cooperation and communication, the effort they invest into translation exercises, the importance they attach to these exercises, and the effectiveness of translation technology in collaborative translation. We use questionnaires to obtain a comprehensive overview of the translation process of and feedback on collaborative translation from student translators. Face-to-face and virtual collaborative translations are performed in English-Chinese translation courses to compare the learning effectiveness of virtual and face-to-face collaboration, enhance our understanding of how collaborative translation facilitates reciprocity, and offer recommendations to enhance translation teaching. This study develops collaborative translation teaching methods and designs collaborative translation curricula. Exploring the core criteria of collaboration, effectiveness, and efficiency through the reports of student translators collaborating face-to-face and online will contribute to the establishment of a collaborative translation framework.
\end{abstract}

Keywords: collaborative translation, translation technology, translator training

\section{Introduction}

Technological advancements have promoted large-scale translation projects that are completed collaboratively by more than one translator. Translators are no longer the stereotyped introverted individuals who work alone and rarely talk to other people; they are now expected to work in teams and participate in discussions. With stronger technologies and communicative practices, the work structures employed by translators today are "hardly conceivable a few decades ago and require different rhetorical skills and communication practices" (Spinuzzi, 2007, p. 266). Currently, translators rely more on technology than their prior generations; they are more flexible and are more likely to be geographically dispersed. Crowdsourcing and fansubbing have emerged as a new research area, and collaborative translation skills have been correspondingly integrated into the

* The article was supported by the Center for Teaching and Learning Development, National Taiwan University, under the Teaching Practice Research Grant. 
training of translators to provide students with opportunities to practice collaboration during translation assignments.

\section{Collaborative translation}

Collaborative translation refers to any type of cooperation between two or more parties involved in translation, including authors, publishers, and translation agencies, to produce a translation. More specifically, collaborative translation can be defined as collaboration between two or more translators to produce one translated product (O'Brien, 2011). Depending on the geographic distance, time zone, or nationality, collaborative translation can occur through various processes.

Traditionally, translators working on large projects work separately on different platforms and combine their translations later, mostly with the help of an editor. This often leads to time-consuming postediting that involves the integration of different styles and word usage into the translation. By contrast, technological advancements have enabled translators to work collaboratively on a single platform, whether on a computer-assisted translation tool such as SDL Trados or online platforms such as Termsoup. Collaborating on a single platform allows translators to contribute to and reuse collective translation memory, provide instant feedback to other translators, and ensure consistency in the final output. Collaborative work on a single platform significantly reduces postediting time and increases efficiency.

To better familiarise students with the increasingly popular trend of collaborative translation, collaborative translation can be integrated into the curriculum through project-based learning (D. Kiraly, 2005). Project-based learning and collaborative learning combined are widely used social constructivist approaches fundamental to tertiary translator training (Robinson \& Olvera-Lobo, 2018). Additionally, Kelly (2005) considers in-class collaborative activities in small groups to be beneficial for student learning. The student-centred learningby-doing method requires students to be active learners and instructors to play more of a supportive role in classes. Studies have found this teaching approach to effectively enhance students' skills beyond translation-specific competencies that are essential for professional translators (Li, Zhang, \& He, 2015; Prieto-Velasco \& Fuentes-Luque, 2016).

Collaborative activities not only make learning more productive and effective but also facilitate interpersonal skill development and social learning experiences among students. Additionally, they enable students to gain confidence and perform strategic actions that are conducive to their ability to work independently (D. C. Kiraly, 1995). Furthermore, during collaborative learning, students are increasingly proactive and are provided opportunities to practice skills such as "problem-solving, reasoning, or justifying proposals and decisions" (Kelly, 2005, p. 102).

Despite the benefits of working collaboratively, translators usually lack tools that enable effective collaboration and discussion with their teammates in real-life 
situations. Frequently, translators work by themselves with shared translation memory on a shared platform. Despite the increase in virtual collaboration activities, virtual collaboration skills have received less attention in the classroom than project-based collaborations. Palumbo and Duin (2018, p. 109) defines virtual collaboration as "a technology-mediated globally dispersed workgroup [of people who] launch, develop, and complete its assigned task."

Face-to-face collaboration differs significantly from virtual collaboration in that virtual collaboration relies heavily on technical communication and familiarity with online tools and environments. Because the digital-native generation is accustomed to information searching and communicating online, they may find virtual collaboration simple. However, studies on collaborative translation have only recently started to fill a gap in translator training and translation pedagogy (González Davies, 2017), and virtual collaboration still remains a promising area of academic research that warrants attention both in terms of technical communication and translation.

\subsection{Translation technologies}

The development of translation technology enables translated texts to be recycled and reused. These advancements not only improve translators' productivity and efficiency but also enhance their translation quality. The translation technology applied in this study is a translation platform that integrates computer-assisted translation tools, a terminology management system, machine translation, and corpus tools. Because this study discusses the use of translation technology in collaborative translation, the technical aspect of translation technology is beyond the scope of this study.

Translation memory constitutes the core of any computer-assisted translation tool that utilises segmentation, automatic search, and fuzzy match functions. During translation, aligned texts of both the source text and target text are simultaneously stored in translation memory for future reuse. When translating similar segments, the system automatically detects saved corpora and displays similar or identical text, highlighting the differences. This helps translators reuse sentences that have been translated before. Furthermore, translators can evaluate the quality of existing translation and perform edits to improve the translation quality.

In addition to translation memory, computer-assisted translation tools feature integrated terminology management systems to help translators save terms that they have searched for before. Freelance translators usually compile terminology databases and translation memory according to different text types or clients. This helps them complete the translation more efficiently when translating texts in similar fields or for the same client. To ensure translation quality, some clients provide translation memory and a terminology database and request the translator to update the two resources upon completion to ensure consistency even when subsequent translations are assigned to different translators. 


\section{Research method}

This study investigates the learning experiences of student translators participating in collaborative translation with and without the use of translation technology through collaborative translation teaching and practical exercises. The effectiveness and efficiency of two types of collaboration (face-to-face and virtual) are surveyed in terms of students' cooperation and communication, the effort they invest into translation exercises and the importance attached to them, the ease or difficultly of applying translation technology, and the effectiveness of translation technology. Face-to-face and virtual collaborative translations are performed in English-Chinese translation courses to compare their respective learning effectiveness, deepen our understanding of how well collaborative translation facilitates reciprocity, and improve translation teaching.

\subsection{Participants}

Participants in this study comprised 27 students from the undergraduate translation and interpreting programme, of which 22 were female students and 5 were male students. These students were mostly junior and senior undergraduate students enrolled in an elective translation course designed to develop students' skills in translating specialised texts, including technical and legal texts. To be accepted into the T\&I programme, the students need to pass a screening test. On average, the students should have an IELTS score of 6.0 or equivalent English proficiency, and they are mostly native speakers of Chinese Mandarin. In this study, the students were divided into groups of three; each group selected one text from one of the fields of journalism, technical news reports, technical texts, and travel texts. They had to select a text piece of their choice to be translated by their peers from English to Chinese Mandarin.

\subsection{Tools}

\subsubsection{Termsoup}

This research used Termsoup for conducting a virtual collaborative translation activity. Termsoup is a cloud-based platform developed by a Taiwanese programmer to accommodate translators' needs. Termsoup is a simple and userfriendly translation platform that provided the participants with experience in translation, user experience, and interface design to increase translation efficiency. Figure 1 provides a screenshot of the Termsoup dashboard where information on the status of documents, deadlines, and translator productivity is provided, thereby facilitating project management and work arrangement. 


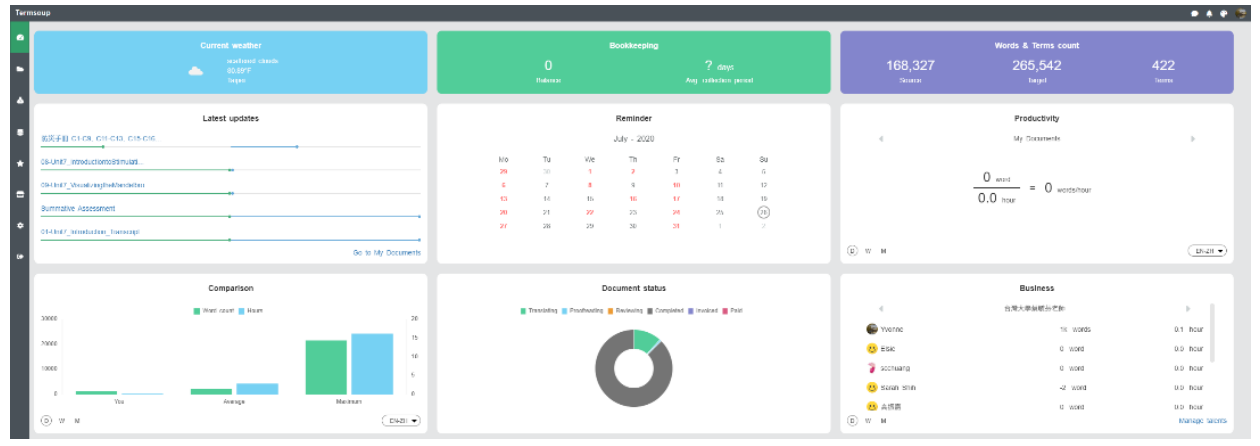

Figure 1. Screenshot of the Termsoup Dashboard

The term query function is one of the most useful functions of Termsoup; it allows terms to be highlighted and searched instantly without the translators having to switch to other webpages. This saves translators a considerable amount of time usually spent on searching and comparing results for the most appropriate translation. Termsoup also stores aligned pairs in translation memory and adds terms to glossaries for future reuse. Machine translation and Netspeak are also available on the same platform (see Figure 2).

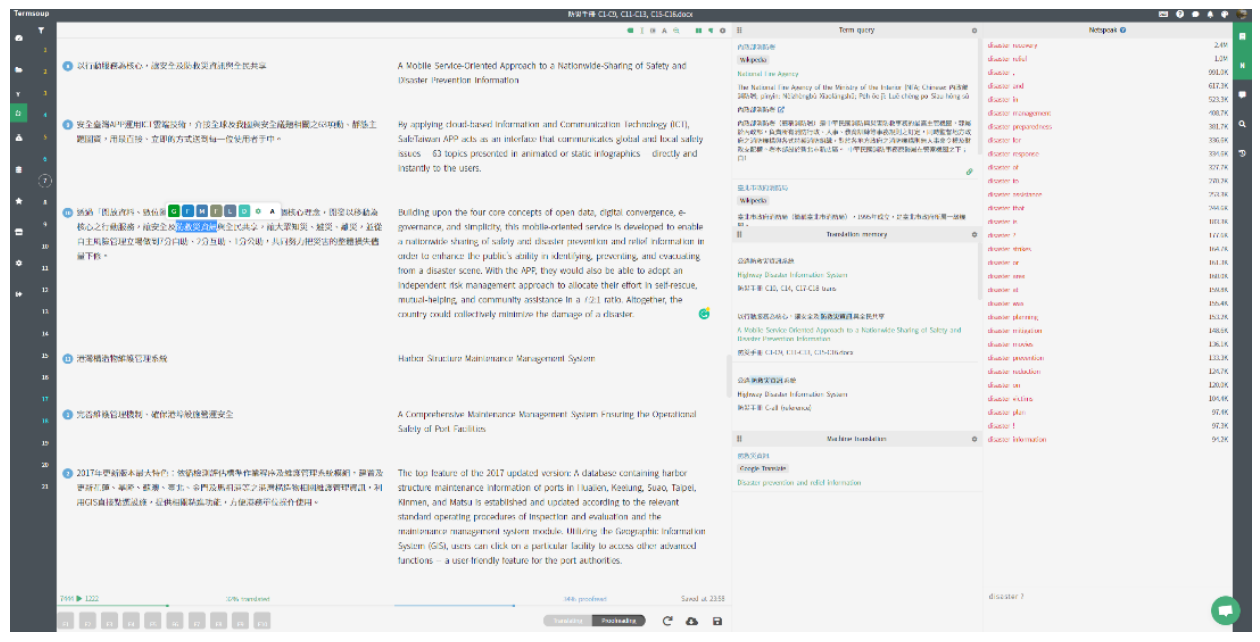

Figure 2. Screenshot of Termsoup's Translation Workspace

During collaborative translation, translators can benefit from the edit history and comment function and leave comments for their fellow collaborators for further discussion 


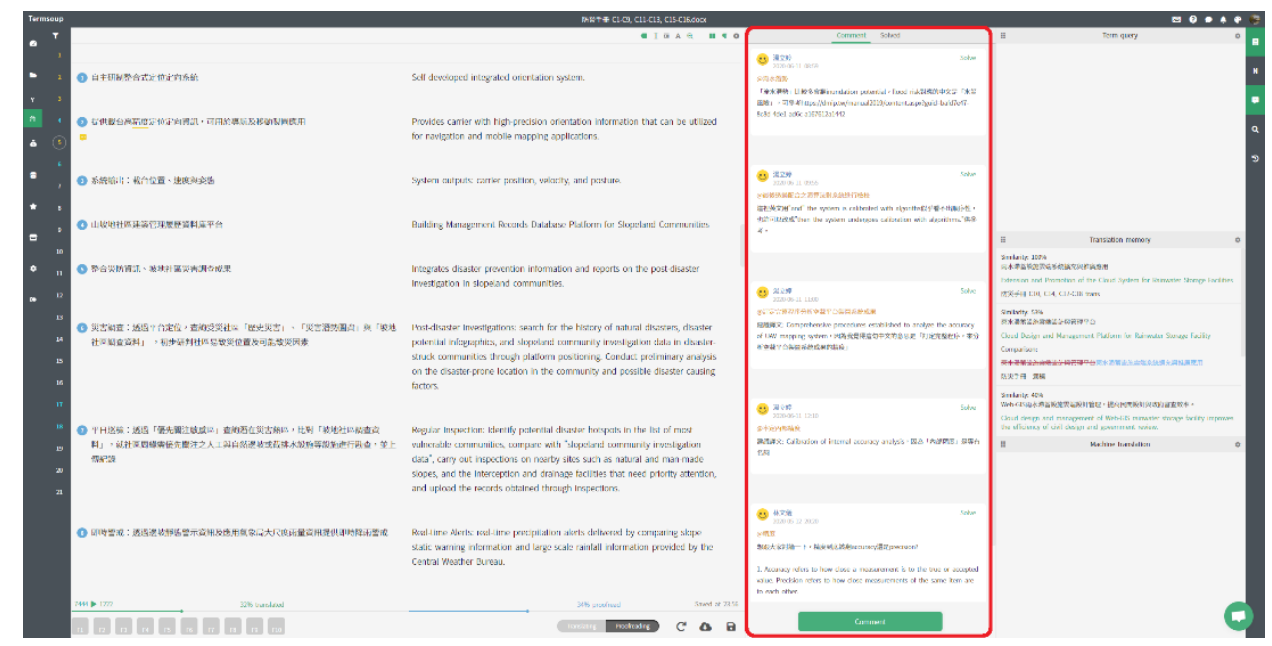

Figure 3. Screenshot of Termsoup's Comment Feature

Termsoup differs from other computer-assisted translation tools because of its user-friendly integrated interface. Unlike some expensive computer-assisted translation tools that can rarely be afforded by novice freelancers with unstable income, Termsoup has a flexible pricing scheme that allows users to use the tool as and when required and disconnect when business is slow. The cloud-based platform allows translators to work without being constrained by the operating performance of their computer systems. Downloading or installing any additional applications is not required. These features make Termsoup the most appropriate introductory computer-assisted translation tool for teaching students and encouraging them to use technology in the translation course.

\subsection{Procedure}

This study was conducted through a five-stage process: 

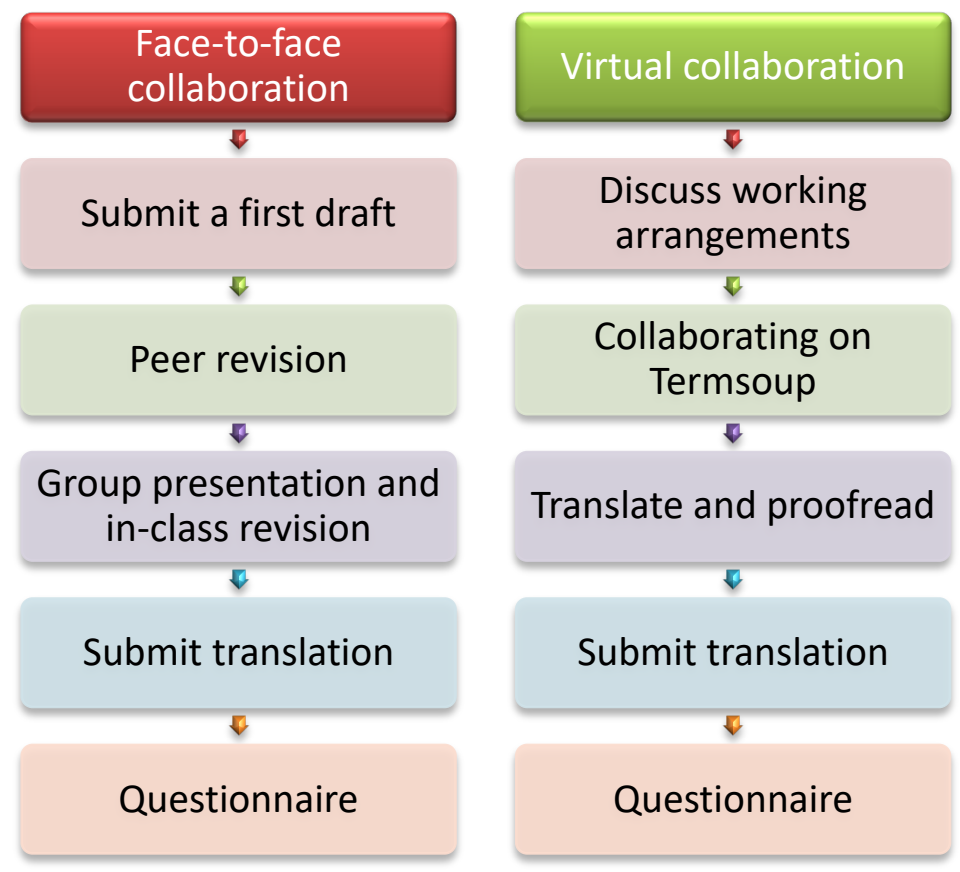

Figure 4. Research Procedure

\subsubsection{Face-to-face collaboration}

Peer revisions and group discussions constitute some of the common classroom activities that promote collaboration in translation classes. Students are usually assigned a piece of translation for review and are expected to give feedback to their peers. This exchange of suggestions and ideas benefits translation students through the shared knowledge and competence of their peers, which in turn improves their translation quality. Therefore, the face-to-face collaborative approach enables students to collaborate through in-person activities.

In this study of face-to-face collaborative translation, the students were required to upload their first drafts to the course website in addition to their final revisions. All student groups selected texts to be translated and analysed the translations of all of their peers. Thereafter, the group members presented their analyses of the source text, typology, and features of the text in question; suggested translation strategies; identified translation difficulties and common errors observed in peer translations and; highlighted their suggested translations during classroom sessions. To provide appropriate and valuable feedback to their peers, translators were expected to consider the accuracy and adequacy of data they collected and discuss their findings with their group members before presenting them before the class. A careful analysis of and targeted research on sources were also performed.

Following the peer revision, the first drafts were peer-reviewed in class. Students were grouped into pairs by the teacher and were asked to use the Track 
Changes function in Microsoft Word to edit and comment while reading the text. Subsequently, the students revised their translation drafts in class according to the recommendations provided during group presentations and peer revision. They were encouraged to discuss the suggestions or clarifications on the translations and peer revisions with their classmates. Each student had 1 hour to revise their translations and were instructed to submit their final revisions before the end of the session.

At the end of the session, the students were asked to answer an online questionnaire analysing the effectiveness of each review activity. The questionnaire included questions to evaluate the usefulness of group presentations and peer revisions, the impact of these activities on their confidence in their translation abilities and language skills, and their learning from the activities. The questions also assessed the difficulties encountered during these activities.

\subsubsection{Virtual collaboration}

At the beginning of the semester, the students were taught how to use translation technology, including computer-assisted translation tools and Termsoup. During the semester, one session was dedicated to virtual collaborative translation, for which the students did not have to attend class but had to complete the task online. Therefore, students had to find alternatives to face-to-face interaction for communicating with their collaborators during the translation process, indicating that preparation was essential for working online.

On the day of the virtual collaborative translation session, the students were given 3 hours to complete a translation of approximately 500 words on Termsoup, regardless of their physical location. They had the liberty to use their preferred communication applications for discussions with their teammates during translation. Additionally, they were encouraged to provide instant feedback to their teammates on their translations during the translation activity and were asked to proofread each other's work upon the completion of the translation.

Subsequently, the students were required to complete an online questionnaire. The questionnaire included questions on the students' experience of using Termsoup, the communication applications used during the translation, students' perceptions of collaborating on a single platform, and difficulties encountered during the translation activity. Furthermore, the students were asked to compare and provide feedback on the effectiveness and their learning experiences of collaborative face-to-face translation and collaborative virtual translation.

\section{Findings}

\subsection{Part one: Face-to-face collaboration}

The results obtained from a preliminary analysis of the online questionnaire indicated that peer revisions facilitated face-to-face collaboration, and that group presentations promoted learning. This is reflected in the feedback from the participants that reported an overall improvement in research, analysis, language, 
and translation skills. By collaborating through group presentations and peer revisions, the students were able to learn from the strengths and weaknesses of their peers' translations.

Preparing for an in-depth analysis of translations can be time-consuming; however, the resources gained through research and shared during presentations can be immensely helpful for students. The carrot-and-stick theory suggests that positive feedback from peers can help students develop more confidence. The key to building translators' confidence, as indicated in the questionnaire, is providing objective and rational feedback supported by research from credible sources. Hence, the students were motivated to search for more information to support their findings, which in turn improved their research skills. In all cases, the participants reported that their confidence improved upon receiving positive feedback from their peers and when they learnt something new.

Comparisons of translations revealed easily detectable differences, and providing a more accurate version of the translation to their peers was often found to be more challenging by students. As expected, about half of the students considered group presentations to be the most difficult but most interesting activity. The emphasis on the correct use of words, grammar, phrasing, and sentence structures during the analysis helped identify areas of improvement and, consequently, improved students' analytical skills and translation quality.

The less interesting peer revisions observed in this study were found to be equally beneficial to the improvement of translations. Positive feedback and practical comments from peers stimulated mutual learning between reviewers and translators. By reviewing and commenting on the translations, the reviewers were able to identify blind spots in the translation and became more aware of how their translations would be viewed by others, which helped them assess their translations more objectively - this required language competence in both directions. By reading and learning from personal and individualised peer revisions, the translators could identify the weaknesses and strengths of their translations and reflect on improving their language and translation skills. Additionally, the students learnt different translation strategies and could adopt words and sentence structures consistently, which helped them correct and improve their translations.

These results confirm that group presentations and peer revisions enable faceto-face collaboration and cooperative problm-solving. The students acquired skills and knowledge required to become an efficient translator, and the translation activity helped create translation memory that can be applied to future translations performed through virtual collaboration.

\subsection{Part two: Virtual collaboration}

To facilitate the study on virtual collaboration, the students were asked to list the advantages and disadvantages of using computer-assisted translation software for producing a target text; this was done to evaluate how well the students had acquired relevant knowledge in computer-assisted translation tools. Among the 
numerous advantages, the students mentioned that computer-assisted translation tools provide translators with a better interface for working with both source and target languages. The students also highlighted the significant features of translation memory and terminology databases, stating that translation efficiency and productivity can be improved through synchronous and consistent translation. Some mentioned that computer-assisted tools facilitate teamwork and collaborative translation, and some reported how the tools benefit translation quality management.

When listing the disadvantages, most students indicated the amount of time required to learn and familiarise themselves with computer-assisted translation tools. The cost of the tool was also identified as a concern. Using these tools may not benefit collaborative translation because all translators would need to have compatible tools for collaboration in the first place. Technical difficulties were one of the common problems encountered when using the tool (see Figure 5), including unstable internet connection and hardware malfunction. Human errors due to unfamiliarity with the tool's features were also deemed disastrous. Additionally, the students highlighted that not all documents benefit from the use of computer-assisted translation tools.

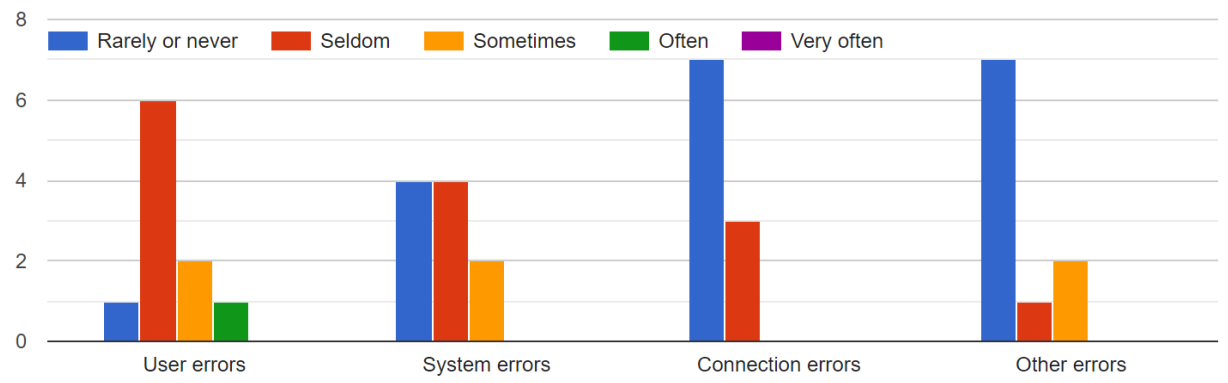

Figure 5. Comparison of User Experience Between Termsoup and Word Processors

Despite the aforementioned disadvantages, when asked how easy or difficult it was to learn using Termsoup, over $80 \%$ of the students agreed that Termsoup was user-friendly. Compared with other word processing software, such as Microsoft Word, over $77 \%$ considered Termsoup to be more efficient for translation. Only three students considered translating using a word processor to be more useful and efficient. 


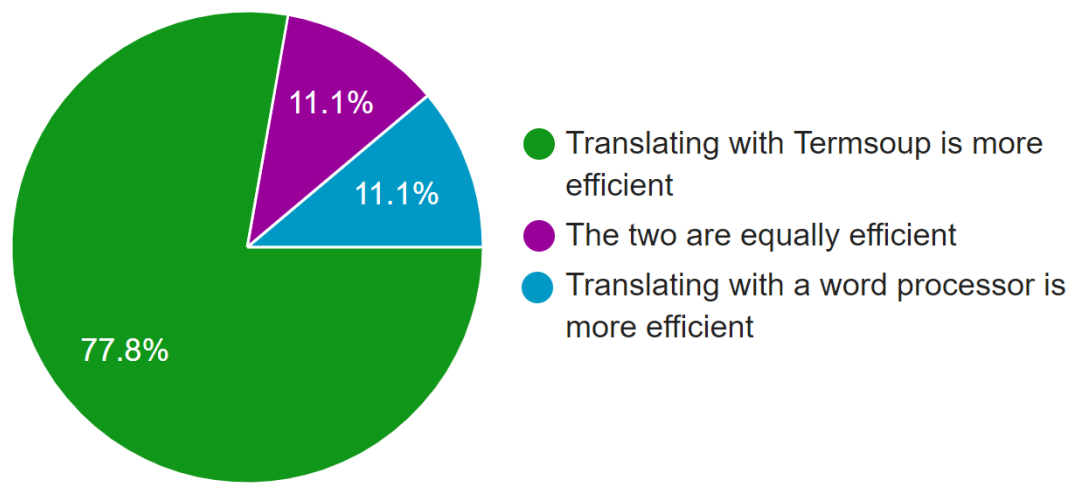

Figure 6. Comparison of User Experience between Termsoup and Word Processor

Because of the time-saving and effective term query function, over $70.6 \%$ of the students considered Termsoup to be helpful for collecting and organising information for the translation assignment. However, surprisingly, $47.1 \%$ of the students did not consider Termsoup to be advantageous over face-to-face mode of work for completing translation assignments. Only $29.4 \%$ agreed with this statement, and $23.5 \%$ held neutral opinions. This finding suggests that the students favour direct, instant, face-to-face communication than complete online collaboration. Nonetheless, if the students were to collaborate online, $69.3 \%$ found that Termsoup effectively facilitated collaboration with other translators for translation purposes.

Coordination and discussions between the translation agents involved are essential for collaboration in the translation process. Over half of the students $(55.5 \%)$ agreed that Termsoup enabled better participation of group members in translation assignments, $33.3 \%$ held a neutral view, and only three students disagreed. When asked how they collaborated online, most students mentioned that they focused on performing their share of work and peer-reviewing each other's work. Additionally, the students reported using multiple technologies for exchange and discussion. Notably, although an Add Comment tool is available on Termsoup, providing a chat box for instant messages, most students used Facebook Messenger as their primary means of communication. Only two students used LINE ${ }^{1}$ and the comment function on Termsoup.

\footnotetext{
${ }^{1}$ LINE is a popular messaging app in Taiwan, used by approximately $91 \%$ of the country's population. It is freely available on smart devices and has an increasing market share (Fulco, 2020).
} 


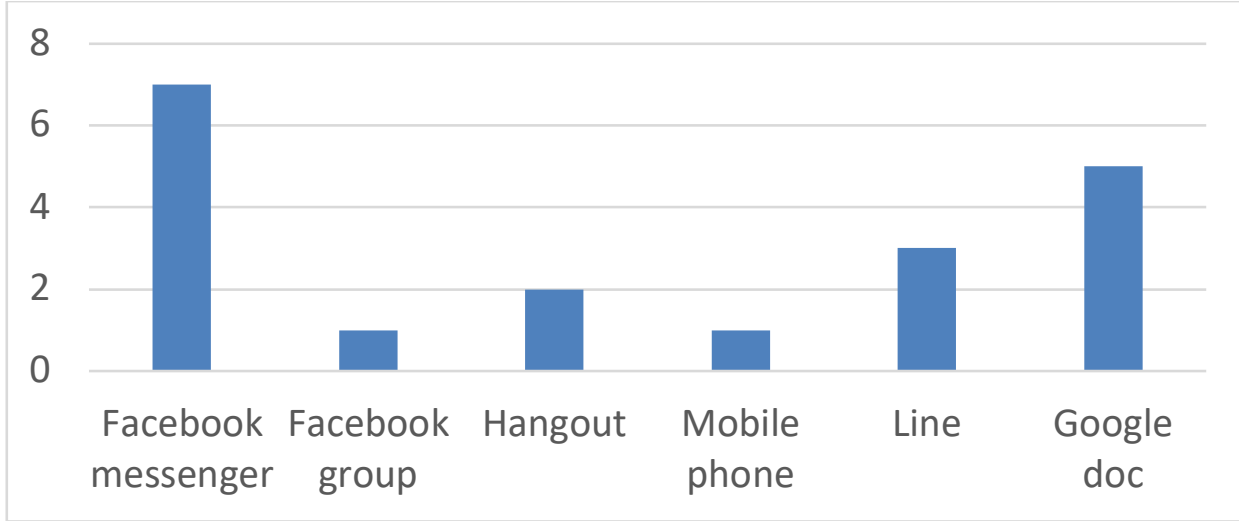

Figure 7. Communication Applications used During Translation

The students who used Facebook Messenger indicated that they could send pictures or make calls whenever required. Additionally, in the case of disputes, Facebook Messenger allows a period of reflection before texting is resumed. However, one student stated that in-person communication is superior to texting, and that taking time to think before responding also affects efficiency. Although Facebook Messenger enables instant communication, in which participants can communicate at the same time, the subsequent conversation may become confusing, and scrolling up and down the screen to seek information can be troublesome. Moreover, using Facebook Messenger to communicate at work may also lead to chatting.

Google Docs was the second most frequently used application in our study. The students favoured the convenience of Google Docs to discuss and communicate directly. Additionally, the application allows multiple people to work on the same document, suggest edits, and add comments, and every change is saved automatically. Moreover, changes can be tracked, thereby enabling users to track the history of changes and determine which changes have been made and by whom. However, when numerous people are working simultaneously, accidental deletions may occur, or texts, which move while other users are typing, may be difficult to read. Additionally, users not signed into the platform remain anonymous; hence, other users are unaware of who they are communicating with. Therefore, some students used other messaging applications while working on Google Docs and mentioned that it saved them time while integrating translations produced by multiple people.

\subsection{Further analysis}

The effectiveness and efficiency of the two types of collaboration were verified from students' attitudes towards peer suggestions for revisions. During face-toface collaboration, the students received extensive feedback for revising their translations, and the type of feedback they referred to when revising their final 
draft was observed. The final drafts of student translations were evaluated against the group presentation slides and peer revisions to calculate the number of changes made by the students in reference to each source. The questionnaire also evaluated whether students' translation revisions were based on group presentations and/or peer revisions. The students' perceptions contradicted their own observations in their final translations.

In their responses to the questionnaire, most of the students mentioned that they referred to both the group presentation slides and peer revisions. However, the revisions identified in their translations were based more on the group presentation slides. The students considered the group presentations to provide a complete analysis. Because the presentation group combined the results of 27 translations, carefully examined every word, and reviewed many references, the credibility of the group presentations was considered to be superior.

Few students referenced peer revisions when revising their final translations. However, more students referred to group presentation slides when producing the final version. Some students were more cautious regarding their peers' comments and re-evaluated the comments before addressing them. One student indicated that peer revisions may be subjective and vary from person to person.

In their final versions, some students revised their translations by using their knowledge of word usage and language to improve flow and readability. They relied more on their own judgment as opposed to reviewers' comments. The students revised their drafts to establish clarity and word consistency, remove typos, and make enhancements to the meaning primarily. Individual students reported having misunderstood the source text and therefore having to revise the subsequent mistranslations. Terminology, conjunctions, and text fluency were also double-checked. Individual sentences were rephrased to ensure that they closely adhered to the rules of the Chinese Mandarin language.

During virtual collaboration, students translate and proofread directly in virtual environments. Although the proofreading process in virtual collaboration resembles the peer revision process in face-to-face collaboration, in our study, intuitive edits increased the number of revision suggestions, with $82.4 \%$ of the students mentioning that their translation contributions were edited or commented on by other group members during the translation process. In face-to-face peer revisions, students tend to be more cautious regarding the credibility of their suggestions. By contrast, instant feedback shortens translators' decision-making processes, allowing more changes to be made to the translation because translators can adopt suggestions quickly.

During collaborative translation, students are expected to engage in languagerelated discussions, provide and respond to immediate feedback on their translations, consider the acceptability and usability of terms and expressions, and make translation-related decisions regarding matters such as consistency and register. Researchers consider inconsistency or mistranslation of terms (Hartley, 2009 , p. 113) as major risks faced by translation in teams. In a virtual environment, students can further benefit from the increased application of technologies (that 
is, computer-assisted translation tools) in machine translation projects. However, the virtual environment may pose some drawbacks.

Some of the drawbacks reported by the students included inefficient communication, team members going offline during the translation, and difficulty in tracking translation progress. When everyone is working online, inefficient communication can create obstacles. Sometimes, feedback and responses to discussions may be less immediate than expected. Tracking translation progress is also difficult when all involved are working on the same file simultaneously. Moreover, increasing the involvement of less active students requires additional intervention. Making compromises when arriving at translation-related decisions is also an important lesson that the students learnt. The students learnt that they should be more accepting of other peoples' opinions and mistakes and be more willing to implement recommended changes. However, the benefits of collaborative translation in the virtual environment outweigh the drawbacks. The opportunities created for students to work efficiently in groups provide an exceptional learning atmosphere that is conducive to the exchange of opinions and critical perspectives on new translation models.

\subsection{Other findings}

To evaluate collaborative translation, the students were asked whether they preferred to work independently or collaboratively, and over half of the students responded that they preferred to work collaboratively. Some students mentioned that their preferences changed because they found that working collaboratively reduced their workload and that they benefitted from others' revisions. However, a few students preferred working independently, citing that they did not need to consider their peers' translations.

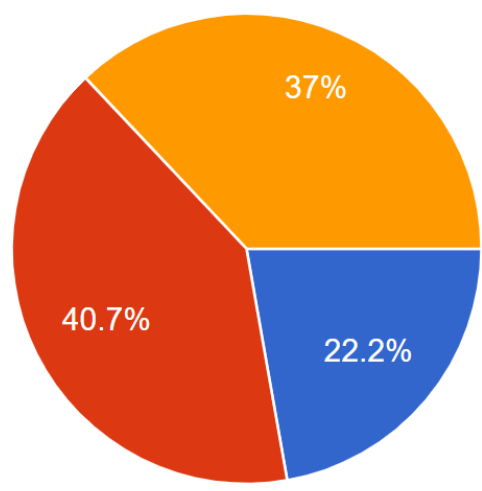

I prefer to work alone

I prefer to work collaboratively

Both are the same to me

Figure 8. Working preferences 
When asked whether they prefer learning through computer-assisted translation tools in a virtual classroom or face-to-face teaching, $40 \%$ of the students mentioned that they preferred virtual classrooms, and $30 \%$ preferred face-to-face teaching, and 30\% found no difference between the two. However, the students mentioned that working and collaborating in a virtual environment increased their awareness of the significant role of technology in translation. Technology offers the ability to choose translation tools on the basis of their needs, and computer-assisted translation and terminology management tools enabled increased knowledge of the translation process. The limitations of these tools were also highlighted.

This study is in line with the updated Wheel of Competence proposed by the framework of the European Master's in Translation (EMT Board, 2017). Face-toface collaborative translation provides students with language and translation competency, personal and interpersonal skills, and service provision, whereas virtual collaborative translation equips students with technological competence. These five areas of competence are considered critical to translator education and training.

\section{Conclusion}

This study was designed to determine the learning experiences of student translators in face-to-face collaborative translation and virtual collaborative translation settings through project-based learning. The results highlight that the students appreciated feedback from group presentations because the credibility of the information thus received benefits from thorough analyses supported by extensive research. The usefulness of feedback was also evident in the students' final translations, in which most students revised their translations according to the suggestions from the group presentation slides.

Students held a positive view of virtual collaboration on a single platform. Over half of the students agreed that Termsoup enable collaborative translation, and over $77.8 \%$ believed that using Termsoup is more effective in translation than word processing software such as Microsoft Word. Observations on the translation process and the final translations produced from virtual collaborative translation indicate that virtual collaboration results in more revision suggestions than face-to-face collaboration does and that most of the suggestions are accepted by students.

Compared with face-to-face encounters, online communication and collaboration tools provide an additional dimension. The use of social networks enabled the students to quickly learn how to proficiently use a platform, which in turn had long-term benefits for their professional lives. Therefore, students should be encouraged to exploit such tools and professional software.

The research findings offer insights into the integration of collaborative learning and translation processes into the curriculum to prepare students for the roles of translators, editors, quality checkers, and project managers and to safeguard student translators' employability. Project-based learning activities 
pose realistic problems that require students to take on various roles and accept collective accountability for the task's final success or failure. Such activities are ideal for preparing translation students for the rapidly evolving work in their field. The insights gained from this study may help translator educators develop collaborative translation teaching methods, collaborative translation curriculum design, and collaborative translation teaching materials.

This study has some limitations. First, the time allocated to the students to familiarise themselves with translation technology and collaboration on Termsoup was limited. The small sample size is another limitation. However, although the current study is based on a small sample of participants, the findings suggest that students are more open to change in a virtual environment, and that subjectivity may influence face-to-face decision-making for translation-related challenges. Despite its limitations, the study provides an in-depth comparison between faceto-face and virtual collaboration in the context of professional translation courses. Further studies evaluating students' provision of and responses to feedback in a virtual collaborative translation project are warranted.

\section{Reference}

EMT Board. 2017). European Master's in Translation - Competence Framework 2017.

Fulco, M.atthew. (2020, May 22, 2020). Japan's LINE Banks on Fintech in Taiwan. Taiwan Business Topics. Retrieved from https://topics.amcham.com.tw/2020/05/japans-line-banks-onfintech-in-taiwan/

González Davies, Maria. 2017. A collaborative pedagogy for translation. In L. Venuti (ed.), Teaching Translation. Programs, Courses, Pedagogies, 71-78. London, New York: Routledge. https://doi.org/10.4324/9781315623139-9

Hartley, Tony. 2009. Technology and translation. In J. Munday (ed.), The Routledge Companion to Translation Studies, 106-127. London, New York: Routledge.

Kelly, Dorothy. 2005. A Handbook for Translator Trainers: A Guide to Reflective Practice: St. Jerome Publishing.

Kiraly, Don. 2005. Project-Based Learning: A Case for Situated Translation. Paper presented at the For a Proactive Translatology - Proceedings, Montréal.

Kiraly, Donald. C. 1995. Pathways to Translation: Pedagogy and Process. Kent, Ohio and London: Kent State University Press.

Li, Defeng, Zhang, Chunling, \& He, Yuanjian. 2015. Project-based learning in teaching translation: students' perceptions. The Interpreter and Translator Trainer, 9(1), 1-19. https://doi.org/10.1080/1750399X.2015.1010357

O'Brien, Sharon. 2011. Collaborative translation. In Y. Gambier \& L. V. Doorslaer (Eds.), Handbook of Translation Studies, Vol. 2, 17-20. Amsterdam/Philadelphia: John Benjamins Publishing Company.

Palumbo, Giuseppe and Duin, Ann Hill. 2018. Making Sense of Virtual Collaboration Through Personal Learning Networks. In M. Birthe, V. Sonia, A. Elisabet, \& M. Bruce (Eds.), Multilingual Writing and Pedagogical Cooperation in Virtual Learning Environments (pp. 109-136). Hershey, PA, USA: IGI Global. https://doi.org/10.4018/978-1-5225-41547.ch005

Prieto-Velasco, Juan Antonio, and Fuentes-Luque, Adrián. 2016. A collaborative multimodal working environment for the development of instrumental and professional competences of student translators: an innovative teaching experience. The Interpreter and Translator Trainer, 10(1), 76-91. https://doi.org/10.1080/1750399X.2016.1154344 
Robinson, Bryan. J., and Olvera-Lobo, M. Dolores. 2018. Preparing Trainee Translators for the Job Market: Social Network Analysis of Teams at Work. In M. Birthe, V. Sonia, A. Elisabet, and M. Bruce (eds.), Multilingual Writing and Pedagogical Cooperation in Virtual Learning Environments, 137-167. Hershey, PA, USA: IGI Global. https://doi.org/10.4018/978-1-5225-4154-7.ch006

Spinuzzi, Clay. 2007. Technical Communication in the Age of Distributed Work. Technical Communication Quarterly, 16, 265-277. https://doi.org/10.1080/10572250701290998

\section{Bio}

Yvonne Tsai is an Associate Professor in the Department of Foreign Languages and Literatures at National Taiwan University. She received her MA in Translation and Interpreting from the University of Bath and her Ph.D. in Translation Studies from Newcastle University. Her major research interests include specialized translation, translation technology, and translation pedagogy. 